\title{
Mouvances Francophones
}

$$
\text { Volume 3, Issue } 12018
$$

Fictions des origines

\section{Problèmes de traduction- d'individuation dans les Fous de Bassant}

Servanne Woodward, Université de Western

\author{
Ontario
}

swoodwar@uwo.ca

https://doi.org/10.5206/mf.v3i1.1685 


\section{Problèmes de traduction-d'individuation dans les Fous de Bassant'}

Telle qu'abordée par Pierre Bourdieu (Les règles de l'art 1992)² dans le domaine littéraire, l'idée d'individuation (à la confluence des institutions et habitus) est utilisée ici pour définir quelques aspects du processus de traduction dans Les fous de Bassan d'Anne Hébert, ${ }^{3}$ où le drame d'une communauté anglophone s'exprime en français. Nous avons ici au niveau de la constitution linguistique du roman un élément de « disparation », pour reprendre un terme de Gilbert Simondon (1989), indiquant que l'individuation (la divergence réversible de l'être migratoire ou sédentaire pour les colonies dans l'exemple du roman d'Hébert) est en devenir, et issue des tensions de l'association d'éléments disparates incompatibles — «Si cette disparation est trop grande, l'action est impossible ${ }^{4} . »$ La disparation comme combinaison des structures potentielles des expressions culturelles linguistiques anglaises et françaises produisent une tension identitaire que le retour apparemment homogène de la traduction du roman en anglais problématise..$^{5}$ La marginalité calculée de Stevens, qui demeure dans le flou représentationnel, donne un statut particulier au rapport « étranger » ou hétérogène de l'individuation, tant au niveau du lexique textuel, qu'au niveau des caractéristiques composant le personnage. Ce protagoniste principal est revenu étranger au point que son frère jumeau ne le reconnaît que lorsqu'il décline son nom. Il se complaît dans posture aliénante pour la société avec laquelle il cultive l'antagonisme. La «traduction » filmique d’Yves Simoneau $(1987)^{6}$ confirme certaines de ces caractéristiques.

\section{Une identité textuelle à la croisée des genres}

Si nous suivons les divers mouvements qui structurent les champs littéraires pour caractériser Les fous de Bassan, tels que décrits par Bourdieu et concernant le tournant du dixneuvième siècle, nous pouvons trouver qu'il s'inscrit dans cette écriture une tendance à l'art pour l'art (peut-être renforcée de Structuralisme), avec une tentation Naturaliste si nous considérons que l'intrigue du roman semble émaner du paysage. La race, le milieu, le moment, ou plutôt pour s'affranchir du vocabulaire Zolien, la généalogie, les lieux et le moment historique dédouanent les personnages du poids moral de leurs actions. C'est le vent qui est responsable du double meurtre selon Stevens :

\footnotetext{
1 Suggestion de citation pour cet article : Servanne Woodward, « Problèmes de traduction-d'individuation des Fous de Bassant» dans Memorias de la VI Conferencia Cientifica Internacional de la Universidad de Holguin, Holguin: Editorial Universitaria, 2013. ISBN 978-959-16-2138-2.

Reproduit ici par l'autorisation de Vilma Páez Pérez, Profesora-Investigadora Centro de Estudios sobre Cultura e Identidad Universidad de Holguín, CUBA (Conference proceeding 2013, WEFLA)

2 Pierre Bourdieu, Les règles de l'art. Genèse et structure du champ littéraire, Paris, Seuil, 1992, $480 \mathrm{p}$.

3 Anne Hébert, Les fous de Bassan, Paris, Seuil, 1982, 249 p. Désormais, les références à cet ouvrage seront indiquées par le sigle $F B$, suivi du folio, et placées entre parenthèses dans le texte. ${ }^{4}$ Gilbert Simondon, L'Individuation psychique et collective, Paris, Aubier, 1989, p. 209 de 293.

${ }^{5}$ Cet article se reportera à la traduction d' Anne Hébert, In the Shadow of the Wind, traductrice Sheila Fischman, Toronto, Stoddart Publishing, 1983, 184 p. Désormais, les références à cet ouvrage seront indiquées par le sigle $S W$, suivi du folio, et placées entre parenthèses dans le texte.

${ }^{6}$ Les fous de Bassan, réalisation Yves Simoneau, production Justine Héroux, scénario de Michel Beaulieu, Sheldon Chad et Yves Simoneau (approuvé par Anne Hébert), Stevens Brown jeune étant joué par Steve Banner, Jean-Louis Millette incarnant Stevens Brown vieilli.
} 
Tout le monde dans la région est d'accord pour assurer qu'il n'y avait pas de vent ce soir là et que a mer n'avait jamais été aussi paisible. Et pourtant, moi, Stevens Brown, fils de John Brown et de Bea Brown, j'affirme que subitement quelque chose s'est rompu dans l'air tranquille, autour de nous....Le vent retrousse....Le vent me fouette.... (FB, 243244)

D'ailleurs le révérend corrobore : «Dans toute cette histoire il faudrait tenir compte du vent, de la présence du vent, de sa voix lancinante dans nos oreilles, de son haleine salée sur nos lèvres » $(F B, 26)$. Les causalités viennent de côtoiements externes disparates (l'île, le vent, la colonie implantée sur le rivage), ce qui n'empêche pas des moments d'introspection «psychologiques ». Nicolas Jones se sait deviné en train de faire des effets de voix pour séduire les petites Atkins (FB, 29, 30). Jones se perçoit en même temps que les autres membres de sa communauté comme omniscient. Chacun expose son sentiment et sa perspective émotionnelle concernant les mêmes événements, un avantage généralement réservé au roman épistolaire. Ici des lettres et journaux sont accumulés en un dossier pour détective. Marilyn Randall a noté qu'à l'examen du dossier, le meurtre n'est pas strictement attribuable à Stevens ${ }^{7}$. Le révérend ou Perceval restent aussi bien suspects. Par ailleurs, l'« Avis au lecteur» nous dit qu'il s'agit de « souvenirs » mais « fondus et livrés à l'imaginaire » $(F B, 9)$. Le journal de Nora et celui d'Olivia surtout, donnés post-mortem, font entorse à l'effet de réalisme, tout comme la langue poétique-littéraire de Stevens. Par conséquent, la trame narrative reste souple ne cernant pas précisément les évènements ni les personnages.

Puisqu'à cause de la position esthétique du texte, la position morale fait défaut, une lecture de tendance féministe ne tient pas comme le remarque Randall : «....l'évidence textuelle réduit ce féminisme inconscient à peu de chose ${ }^{8}$ », et le discours « viril », à la fois misogyne et poétique de Stevens en psychopathe pourrait passer pour lucidité et violence libertine, contre ce que Bourdieu caractérise par les « saines passions de l'amour pour la famille et la société » défendues par Emile Augier en son temps ${ }^{9}$. De toute évidence le sadisme misogyne n'équivaut pas à une libération corporelle, sexuelle, ni à une libération psychologique, pas même pour les hommes.

Les femmes au foyer ne semblent pas avoir un sort particulièrement enviable et dans l'agression de Stevens contre Olivia, à qui il veut faire avouer sa sexualité, il semble y avoir des relents de «l'horreur du bourgeois » ${ }^{10}$. S'il fallait caractériser ce roman, ce serait du côté de la parodie amicale de George Sand par Flaubert dans L'éducation sentimentale ${ }^{11}$. Et cependant, Hébert y apporte une modification importante. Il ne s'agit pas de « bien écrire le médiocre ${ }^{12}$ ", car la prose est trop poétique et les personnages trop originaux dans leur isolement et l'effet esthétique rabaisse « l'intérêt dramatique ${ }^{13}$. Hébert ancre l'identité de

\footnotetext{
7 Marilyn Randall, «Les énigmes des Fous de Bassan : Féminisme, narration, et clôture », Voix et Images, vol. 15, no. 1 (43) 1989, p. 80 de p.66-82.

URI: http://id.erudit.org/iderudit/200817ar

DOI: 10.7202/200817ar (page consultée le 30 avril 2014).

8 Marilyn Randall, «Les énigme », p. 67.

${ }^{9}$ Pierre Bourdieu, Les règles de l'art, p. 117.

${ }^{10}$ Ibid., 119.

${ }_{11}$ Ibid., 120. Gustave Flaubert, L'éducation sentimentale, histoire d'un jeune homme, Paris, Michel Lévy frères, 1869, 2 vols. in- $8^{\circ}$ de 427 p. et 331 p. respectivement.

${ }_{12}$ Pierre Bourdieu, Les règles de l'art, p. 140.

${ }^{13}$ Ibid., 155.
} 
son texte dans un va et vient entre la culture anglophone d'Amérique du Nord et la tradition littéraire française, entre le familier et l'étranger, une position qui rappelle les mouvements caractéristiques de Stevens.

\section{Sens en translation}

Les fous de Bassan accuse une tendance à évoquer des titres anglophones. En dehors des citations identifiées en exergue de chapitre, citations qui servent à caractériser les personnages, à orienter la lecture de leur témoignage, nous avons des phrases qui font remonter d'autres auteurs à la mémoire : «Words, words, signifying nothing» (FB, 46), en anglais dans le texte, se réfèrent évidemment à la scène v, acte v, de Macbeth, où les mots perdent leur sens et s'assemblent en vagues de bruits. Moins évident : «On pourrait croire que le jour n'aura pas lieu » $(F B, 50)$, évoque peut-être « la guerre de Troie n'aura pas lieu ${ }^{14} \gg$ où il est question de séductions et des guerres qui s'en suivent; «Trop de bruit et de fureur depuis mon enfance » rappelle le « sound and fury » de Hamlet plutôt que la traduction « too much noise and furor ever since childhood » $(S W, 180)$. Le fait que la citation ne soit pas directement en anglais dans le texte d'Anne Hébert peut avoir motivé la traduction non Shakespearienne. Ronald Ewing a lui aussi pensé à The Sound and Fury ${ }^{15}$, incluant les étranglements convulsifs et «innocents » de Benjy, qui évoquent un principe de criminalité sans responsabilité pour le personnage de Faulkner, comme pour Stevens et Perceval entre autre. En effet, les actes d'agression contre les cousines du roman d'Anne Hébert sont causés par les facteurs externes disparates et constitutifs de la communauté. Le vent, le Nord comme entité économique (le village vit de pêche).

Enfin, «Me voici donc de retour au pays natal » peut évoquer «Cahier d'un retour au pays natal », le poème d'Aimé Césaire publié en Août 1939, en vers et prose et concernant le thème de l'identité culturelle des noirs d'Afrique sous la colonisation. Ce qui fait verser le texte de la petite communauté de Griffin Bay vers des contextes éclectiques plus larges, où il est question de gouvernement et de lignages déliquescents (avec Shakespeare), ou bien de colonialisme.

D'ailleurs, un passage permet à Stevens de se comparer directement aux noirs américains du Sud des Etats-Unis dans une lettre datée du 26 juin 1936, lettre qu'il envoie à Michael Hotkiss à qui il vient de mentionner la Floride et la Géorgie : «Il n'y a que les nègres qui font aussi mauvais effet, postés là, au bord de la route en plein soleil, dans leurs vêtements déteints, immobiles, pareils à des statues, seuls ou par petites familles, attendant un lift comme on espère le Paradis » $(F B, 57)$. Le mot «lift» en anglais dans le texte indexe la langue de la lettre de Stevens comme issue du Québec, et de la jointure entre le français qui a naturalisé l'anglais ambiant- tout comme la nature ambiante et le vent a naturalisé et façonné les habitants de Griffin Bay. Le thème d'un côtoiement des communautés francophones et anglophones et de ces nègres en attente de mouvement évoque le titre des Nègres blancs d'Amérique, de Pierre Vallières, livre qui en 1968 est devenu un classique du mouvement de libération et indépendantiste québécois ${ }^{16}$. La date même de la lettre, qui évoque des noirs particulièrement statiques en bordure de route, est aussi celle où Jesse Owens établit le record du 100 mètres aux Jeux olympiques de Berlin (10.2), record ayant

${ }^{14}$ C'est le titre d'une pièce de Jean Giraudoux dont la première fût jouée le 22 novembre 1935.

${ }^{15}$ Ronald Ewing, "The English World of Griffin Creek », Canadian Literature, vol. 105, été 1985, p. 100-110.

${ }^{16}$ Pierre Vallières, Nègres blancs d'Amérique, autobiographie précoce d'un «terroriste » québécois, Montréal, Éditions Parti pris, 1967, 402 p. 
particulièrement détonné par rapport à l'idéologie fasciste environnante, puisque le champion de ce titre était indéniablement noir. L'être stationnaire sédentaire égale ironiquement le mouvement record du championnat de vitesse humaine par un noir au moment où les noirs sont statiques au bord de route, «immobiles comme des statues »largués économiquement et socialement parlant. Cette phrase passe parfaitement à la traduction « still as statues », par contre, «waiting for a lift the way others might hope for heaven » (SW, 41) ne rend pas l'indice francophone québécois qui englobait l'anglophone du et le francophone du Nord avec les parias noirs. La traduction anglaise rapporte les anglophones de Griffin Bay à leur langue de fiction propre. La caractéristique de « nègre blanc » adoptée par les québécois indépendantistes pour dénoncer le colonialisme de l'économie angloaméricaine se retourne sur un membre de cette communauté Nord anglophone en occultant la dimension Québécoise.

Ainsi, le schisme économique et linguistique des deux communautés concurrentielles de la colonisation d'Amérique du Nord est nivelé entre anglophones et francophones par l'usage unique de l'anglais. Dans la version française, noirs, anglophones et francophones sont tous susceptibles de voyager comme de s'enliser en bord de route, dans les aléas et fluctuations qui affectent les peuplements coloniaux du continent. A la traduction anglaise, les trois groupes perdent en solidarité en tant que victimes identiques des revers économiques et politiques ambiants. Car dans la version originale française les principes d'individuations d'une langue l'autre ont tendance à s'effondrer lorsque une langue est aussi bien une autre. Le roman était donc déjà écrit en «traduction » française dès sa conception, et cependant le retour vers l'anglais accuse un décalage.

\section{Retour à l'Anglais}

Une phrase nominale introduit Les fous de Bassan: «La barre étale de la mer, blanche, à perte de vue, sur le ciel gris, la masse noire des arbres, en ligne parallèle derrière nous » ( $F B$, 13). Elle donne à voir un paysage statique, presque abstrait. Son mouvement est encore imperceptible, et cependant, l'admirable traduction anglaise de Sheila Fischman nous livre déjà sa dynamique : " $A$ strand of sea poised between tides, white, as far as the eye can see, and against the gray sky, in a parallel line bebind us, the black bulk of trees » (SW, 9). Il s'agit bien d'un paysage qui se resserre en étau sur la petite communauté de deux cents ans, mais nous l'apprenons une quinzaine de pages plus loin : "Je me désagrège à petit feu dans une demeure vermoulue, tandis que la forêt, derrière moi, se rapproche de jour en jour, de nuit en nuit, plante ses pousses de bouleau et de sapins jusque sous mes fenêtres » (FB, 27). La mer en étendue blanche devant une masse d'arbres noirs peut représenter l'espace de l'écriture à venir à l'ouverture du roman, alors que plus bas il s'agit d'une blancheur d'ensevelissement et d'oubli sans postérité. Dans la traduction anglaise, « against the gray sky» traduit mal « sur le ciel gris », la préposition «sur » étant déviante par rapport à ce qui serait attendu (" sous » par exemple). D'un côté la forêt qui gagne, et de l'autre, la mer qui engloutit la terre à chaque marée, celle qui appelle dans ses flots la matriarche de la communauté et deux de ses jeunes favorites. Olivia ayant rejoint ses aïeules défuntes, ces dernières cherchent à l'empêcher de retourner hanter le village et les lieux de sa passion pour son meurtrier.

Une allusion au schisme politique et culturel qui sépare le révérend des " papistes » $(F B, 13)$, reste le seul antagonisme sensible qui subsiste à la traduction anglaise contre les francophones qui sont en majorité d'allégeance catholique. Nous quittons là le domaine culturel de la littérature française pour entrer au point de friction entre les cultures anglophones et francophones d'Amérique du Nord. En effet, l'opposition irlandaise et anglaise est attisée de façon notoire par ce schisme Protestant/Catholique, et jusqu'à date, le 
seul Catholique à avoir réussi à devenir président des Etats-Unis reste J. F. Kennedy. Mais que ce conflit surgisse en termes de papisme dans un texte rédigé en français atteste du fait que cette expression française est traversée d'un conflit typiquement anglais, d'un contexte anglais. Lorsque tout le texte passe à l'anglais, le face à face franco-anglais est amoindri, le « papisme » devenant un élément étranger parmi d'autres.

La phrase dénotant l'impatience du révérend vis-à-vis des « papistes » « m’emplit les yeux de lueurs fauves stridentes » $(F B, 13)$ —, fait allusion à un antagonisme naturalisé, viscéral, et il correspond également à un système de couleur parcimonieux qui caractérise le paysage comme le personnage. Tout d'abord parce qu'étant fils de familles «Jones » (évoquant le « jaune » pour une prononciation à la française) et «Brown » (désignant le «brun »), il utilise une palette jaune et brune pour se peindre lui-même, puis ses douze générations d'ancêtres masculins : « neige jaune », « roux », «blond », « châtain » $(F B, 15)$. Ainsi, la traduction de «fauve » par « savage » [sauvage] $(S W, 10)$ est certes justifiée, mais elle estompe l'opposition entre les bruns-jaunes et le village voisin, aux « bicoques peinturlurées en rouge, vert, jaune, bleu, comme si c'était un plaisir de barbouiller des maisons et d'afficher des couleurs voyantes» $(F B, 13)$. Le multicolore vif est opposé aux couleurs ambiantes de Griffin Bay qui sont aussi celles de l'individuation du personnage du révérend. Il s'agit d'une structure non synthétique de l'hétérogène. Ici, le personnage se compose de couleurs restreintes conformes au paysage, et de couleurs rapportées, peut-être plus conformes aux couleurs des poissons et des fonds marins, où sont allé se réfugier les femmes du lignage ancestral de Griffin Bay. Des couleurs, tels les lambeaux de robe rose de Nora, permettront d'identifier le morceau de son tronc humain que la mer ramènera sur la plage.

\section{La guerre des genres et des couleurs}

Janis L. Pallister s'attache à la sauvagerie des oiseaux et des griffons qui imprègne les caractérisations des personnages de Griffin Bay, notant entre autre que les jumelles ont " griffonné » les portraits $(F B, 48)^{17}$. Ainsi, lorsque les jumelles ont terminé de peindre les femmes de la galerie de portraits du révérend, elles ont fait une débauche de couleurs, et elles en ont tâché leurs vêtements — le multicolore dépeint également les papistes et les femmes. Devant l'évidence de leur plaisir de peindre et de colorer, le révérend juge opportun de leur interdire désormais l'usage des couleurs comme l'entrée de cette galerie $(F B, 16,18)$. Le multicolore s'oppose à la « frugalité » $(F B, 17)$ fondatrice et patriarcales du jaune-brun. Si les jumelles de patronyme «Brown» restent dans l'ascétisme frugal au point de grandir en jeunes vieilles et vieillir en vieilles jeunes osseuses, sans sexualité ni individualité déterminée, la paire indifférenciée des petites Atkins devient un corps de gémellité non identifiable: «Depuis le début de l'office Perceval a les yeux fixés sur ses deux cousines, Nora et Olivia. Un seul animal fabuleux, pense-t-il, à deux têtes, deux corps, quatre jambes et quatre bras, fait pour l'adoration ou le massacre » $(F B, 31)$. C'est en ces mêmes termes que Stevens Brown décrit les deux filles juste avant d'étrangler et violer: « ....je les tutoie comme une seule et même créature à deux têtes, quatre bras, quatre jambes et deux petits sexes cachés » (FB, 104). Incidemment, ce détail du tutoiement pour une adresse plurielle devient inexpliqué dans la traduction : "I speak to them as if they were a single créature... » $(S W, 75)$. En l'absence d'opposition tu/vous en anglais, le tutoiement tombe de la traduction. Or en français le tutoiement est dévoyé de sa caractérisation du singulier pour deux femmes.

${ }^{17}$ Janis L. Pallister, «Anne Hébert et Guylaine Dionne, deux interprétations opposées des mêmes Fous de Bassan », Women in French Studies, vol. 13, 2005, p. 71 de p. 65-81. 
Il semblerait que mer et mère produisent des lignées féminines désirables mais indifférenciées aux yeux des hommes qui semblent voués à tromper leurs femmes et à les tuer, physiquement et psychologiquement. La mère d'Olivia meurt des coups que lui assène son époux ; Irène, l'épouse du révérend, se pend ; Felicity Jones, la mère du révérend, a développé une franche haine des hommes. Toutes sont trompées, indifférenciées les unes des autres au niveau le plus intime de leur personne, par le désir-pulsion des hommes qui les confondent entre elles en masse. La singularité, la distinction de l'unicité leur est refusée. Elles sont jumelles, plurielles, vouées à la reproduction unilatérale de blond-roux-brun Jaune/Jones/John confondus. Leur corps est saisi non pas dans sa singularité individuelle mais dans sa multiplicité, multitude, capacité de multiplication. Le genre féminin est l'espèce.

Irène s'est soustraite cette économie onéreuse lorsqu'elle « ....fait semblant de dormir la nuit, lorsque je m'approche d'elle....» $(F B, 44)$. Elle ne danse pas. Elle ne voit pas. Elle ne parle pas. Retirée de la reproduction, elle pourrait prétendre à l'unicité jusqu'à ce que le révérend la désire comme il en désire une autre, comme une autre à sa place. Perceval la force à voir cet accident qu'elle préférait ignorer, et elle se pend. Dans cette guerre des hommes et des femmes, le révérend garde un espoir pathétique : «Que seulement, Irène, ma femme, me donne un fils et je l'offrirai à ma mère, Felicity Jones, en témoignage de ma puissance » $(F B, 32)$. Il rêve de donner à sa mère un fils qu'elle aime, elle qui manifestement préfère les filles, rejetant les garçons comme autant d'ennemis du genre féminin (" cochons ", $F B, 75)$. Elle a dû vivre constamment trompée, parce que son époux semblait préoccupé par la performance reproductive, et elle a rejeté sa soif de passion sur la mer, et les promenades nocturnes et solitaires en bordure de plage.

Et cependant, les femmes qui affirment leur désir d'amour réciproque sont humiliées au point d'être anéanties. Ayant refusé de marcher au bras de Stevens, et ayant insulté sa virilité, Nora meurt étranglée, agenouillée dans sa chute mortelle. Faute de terme plus précis, sa voix mêlée au vent de la mer, elle l'avait appelé un " garçon manqué » $(F B, 92)$. La traduction de « sissy» (SW, 66) montre bien qu'elle ne l'estime pas particulièrement viril. Stevens l'est bien suffisamment avec Maureen, qu'il finit par sevrer de sexe, la mettant « à la diète » en allant coucher à la grange pour qu'elle « découvre....sa nouvelle solitude, plus grande que la première » $(F B, 69)$. Alors que « sissy» montre bien le dévoiement de la caractérisation injurieuse de Stevens par rapport aux codes de comportements sexuels réciproques sur lesquels Nora croyait pouvoir compter, le terme ne montre pas que ce mot s'applique généralement aux filles qui n'adoptent pas les restrictions corporelles qui leurs sont octroyées. "Garçon manqué » serait alors plus proche de «Tom boy » que de « sissy ». Le fait qu'elle connaisse cette appellation laisse à penser que son agressivité physique lui a valu cette insulte pour son propre compte. Elle est donc identifiée comme ayant une activité qui dépasse les confins de la féminité. Mais Stevens va plus loin. Il s'agit de " punir» le désir réciprocité de Nora. Le dysfonctionnement des couples finit par dépeupler Griffin Bay. Les femmes meurent, et se réfugient au fond de la mer : « Ma grand-mère est un dauphin » (FB, 71) selon Perceval. ${ }^{18}$ Il annonce à Stevens qu'Olivia a « Un orteil qui est collé à l'autre par une petite peau, comme un canard» $(F B, 81)$. Les personnages féminins ont tendance à la métamorphose. D'ailleurs, La petite sirène de Hans Christian Andersen ${ }^{19}$ est évoquée pour introduire le récit d'Olivia.

18 «My mother is a fish» [« ma mère est un poisson », je traduis] est bien l'ouverture inoubliable du roman As I Lay Dying, de William Faulkner.

${ }^{19} \mathrm{Il}$ s'agit d'un conte de fée où la petite sirène veut suivre l'amant qui venait l'écouter au bord de l'eau, mais pour le suivre sur terre elle doit se couper le corps de poisson pour en obtenir deux 


\section{WORDS, WORDS}

Mais beaucoup plus troublant, et la tendance fut enregistrée par Randall, les personnages se reprennent les uns les autres presque mot pour mot, ou disent avoir déjà dit quelque phrase qu'ils n'ont jamais prononcée, mais que d'autres ont énoncée, et si une quelconque résistance féminine peut être détectée, Nora comme Olivia reprennent pour leur compte les discours dominants qui les assassinent, partageant ainsi une partie de la responsabilité de leur propre meurtre ${ }^{20}$. Les actes qui devaient ouvrir à d'autres états de devenirs pour les personnages les ont en fait mis sur une voie sans issue que la métamorphose ultime des morts. Olivia hante les plages de Griffin Bay de son ombre mêlée à celle de ses ancêtres féminines peuplant le fond des mers. L'individu est une horde, une nuée, un banc, une série. Stevens a vécu la deuxième guerre mondiale dans l'hallucination des corps de femmes qui chutent agenouillées. Le révérend, incapable de procréer, a peint la galerie entière des portraits de ses ancêtres à son image. Ce que Simondon interprétait comme un processus d'émergence de deux réalités disparates nécessaire à l'individuation comme devenir s'effondre dans le piétinement du rapport au même dans la ligné virile. Ni les portraits ne s'intègrent les uns aux autres, ni n'existent-ils dans la tension, l'opposition, la disparité, ni dans la profondeur temporelle historique par rapport au présent. Ce flottement qui hante les portraits du révérend remontant sa généalogie en en inversant l'origine ressemble à l'aplatissement que la traduction anglaise fait parfois subir à la «traduction » initiale du roman en français à partir d'un monde anglophone, flottement qui aboutit selon Randall à « une lecture plurivoque de la narration »

Sans doute en va-t-il de même pour les personnages de l'adaptation filmée de ce roman, déjà parce que Simoneau a réduit le nombre des personnages. Télescopés ensemble, il leur arrive d'échanger leurs phrases. Ainsi, par exemple, c'est Nora, non plus Perceval, qui révèle à Stevens que le pied d'Olivia est palmé. Stevens est rejeté de la communauté masculine à cause des mots étrangers qu'il utilise. Par exemple lorsqu'il dit avoir voyagé au Sud de la Floride dans « les Keys», Bob Allen le lui fait répéter deux fois : « les quoi ?». En dehors de l'aspect comique de l'écho Keys/quoi, l'anglais est abordé de façon circonspecte par les francophones. Lorsque Stevens utilise l'adresse «Boys» il finit par provoquer une bagarre entre les jeunes pêcheurs qui ne savent pas si elle est effectivement utilisée au Sud des «Etats», et par conséquent, qui ne peuvent pas évaluer les paroles de Stevens. Ce qui ressort plus profondément est que Stevens semble peu digne de confiance. Sa parole n'a pas de valeur, jetant des doutes sur les boutades de Stevens vieilli exprimant la raison de son retour (se « libérer de la fureur du vent. »)

Tous les personnages finissent par se fondre dans des colonies et nuées de fous de Bassan. Le révérend écarte les bras parmi eux dans sa prière en bord de mer après le suicide d'Irène ; Perceval a tenté un envol avec une nuée de ces oiseaux, mais il est resté indéniablement cloué au rivage ; Irène a effectué elle aussi un envol en bordure de falaise, mais elle est tombée à pic ; Olivia semblait sensible à l'invitation au voyage de Stevens dans la scène du repassage où il est venu la voir chez elle. Ses compliments et son appel au voyage

jambes avec lesquelles elle puisse le suivre sur terre. Comme elle en perd la voix, le beau jeune homme ne la reconnaît pas et il ne reste plus à la petite sirène qu'à aller se noyer dans l'eau d'où elle n'eût jamais dû sortir.

${ }^{20}$ Op. cit., Marilyn Randall, « Les énigmes », p. 80-81.

${ }^{21}$ Ibid., 81. 
semblent spontanés et systématiques. Mais seul Stevens migre en boucle. Il se fait appeler « cousin-voyageur» lors de sa première rencontre avec Bob Allen et les autres jeunes hommes. Il semblerait que sa compulsion soit de faire partir Perceval ou Olivia avec lui mais sans préconception, ni préparation, ni but précis que de leur permettre d'accompagner son départ.

Une image de fou de Bassan s'intercale à des moments dramatiques de l'action lorsqu'Irène décide de s'envoler de la falaise ; lorsque Stevens complimente Olivia. Olivia a des caractéristiques d'oiseau migrateur si elle observe que c'est sa robe qui est déchirée en bandelettes pour faire les guirlandes de fête - ces dernières ressemblent à autant d'oiseaux à plumes blanches et aux ailes déployées. Le conflit entre les villageois et Stevens jeune proviendrait de l'hypocrisie qu'il leur détecte, de se prétendre sédentaires dans une île de migration, de ne pas reconnaitre leur vraie nature migratoire, leur désir de départ, issue de leur origine et devenir voyageurs tels les fous de Bassan. Leurs moments de rires (les filles qui se baignent), les cris d'Olivia se muent en cri d'oiseau au début et à la fin du film qui rejoue le meurtre. Puisque Stevens vieilli semble enclin à laisser un papier qui bat au vent s'envoler de ses mains, pour un temps, il n'est pas impensable qu'il soit venu plus généralement faire un lâcher de portrait libérateur.

Au lieu de remonter son mécanisme, Maureen bloque le balancier de son horloge après la mort d'Irène. Dans le film, le retour migratoire de Stevens-narrateur évoque une issue de l'effacement du dernier témoin dans les ruines d'une société arrêtée. La chaine narrative est bloquée dans une répétition mortifère. Un instant, l'on pourrait penser que Stevens jeune est revenu chercher ses êtres chers, Perceval à défaut d'Olivia, ou l'inverse ${ }^{22}$. Katrhryn Slott, suggère que le charme sexy de Stevens est celui de la victime du patriarcat ${ }^{23}$ qui se révolte et qui est revenue lutter contre les oppresseurs. Mais elle estime aussi que Stevens vieilli rentrerait sous le joug du patriarcat et de l'église, de la religion et de la famille, en faisant état de son regret tardif quant à une révolte qui s'explique moins par un axiome de liberté que par la tragédie d'un amour non partagé ${ }^{24}$ (from agent p. 22). La beauté du meurtre fait partie d'un certain type de romantisme ou de libertinage, mais encore faudrait-il qu'il s'inscrive dans un absolu de révolte, et non pas en simple perversion d'un besoin ou d'une domination inassouvie. Stevens peut difficilement se résoudre en un libérateur des ombres de sa communauté dans une migration céleste ou économique. Sa pulsion du civet de lapin qui passe par l'étranglement laborieux et incomplet d'une lapine enceinte suivi du viol de Maureen en passage annonciateur du viol et étranglement d'Olivia peut difficilement passer sous les couleurs sympathiques d'une émotivité contrariée, ou d'un désir communautaire. Il semble plutôt être venu se substituer à la position patriarcale centrale, maittre nostalgique au milieu des ombres des disparus.

\section{Images 1987}

Les images qui viennent à l'esprit au visionnement du film de Simoneau rapportent au Romantisme. Les éléments et les rochers austères évoquent ses déserts tragiques et insulaires. Les éclairages et les mises-en-scène du corps et du visage de Stevens évoquent les

\footnotetext{
${ }^{22}$ Elspeth Tulloch, «Yves Simoneau's Rewriting of the Troubled Manhood Script in Anne Hébert's Les fous de Bassan », Essays on Canadian Writing, vol. 76, 2002 Spring, p. 105 de p. 83-116.

${ }^{23}$ Katrhryn Slott, «From Agent of Destruction to Object of Desire : The Cinematic Transformation of Stevens Brown in Les fous de Bassann, Québec Studies, vol. 9, 1989 Fall-1990 Winter, p. 22 de p. 1728.

${ }^{24}$ Ibid.
} 
illustrations de Paradise Lost de Milton. Il s'agit des lumières contrastées et des surexpositions lumineuses qui donnent l'impression que la chevelure blonde est une flamme spirituelle embrasée sur son front. Dans la lumière bleuie à peine tamisée, son corps nu est exposé triomphalement, symétriquement, à la première scène de baignoire, et dans une pénombre de folie qui évoque les cauchemars de Goya après le pugilat de la corde à linges. Son chapeau est apparenté au couvre chef d'un portrait de Goethe qui se repose au cours d'une promenade champêtre. La scène de larmes de Stevens qui se met les poings sur les yeux évoque une illustration du jeune Werther, excepté qu'il s'agit ici de rage selon les sous-titres, plutôt que de désespoir.

Autrement, le film opère de nouveaux décalages : l'uniformité des couleurs jaunes-brun de la généalogie Jones-Brown est dérangée dans le nom de John Brown, devenu "Timothy » Brown. Les portraits qui entourent Stevens Brown vieilli semblent être de son cru. Il a fait des paysages portuaires et marins, des vues de l'île. Il ne semble pas qu'il ait pillé la galerie du révérend mais qu'il ait fait un atelier de peinture de l'église abandonnée. La motivation du retour de Stevens Brown vieilli serait de rectifier la vision de sa mémoire par la projection d'images de son île et de sa parenté comme de lui-même : "Il ne me reste qu'une image déformée de moi-même » annonce Stevens Brown joué par Jean-Louis Millette. Après qu'il annonce qu'il soit revenu pour se « voir comme j'ai toujours voulu me voir, moi Stevens Brown », le visage de Steve Banner apparait.

Visuellement, le personnage reste dissonant voire incohérent : Stevens en homme mûr est un brun au corps massif, au visage expressif et mobile, et aux yeux de charbons dans un visage long; Stevens en jeune homme est un éphèbe blond-blanc, élancé, aux yeux très clairs et au visage carré, figé dans l'expression intense et avide. Puisque Stevens Brown vieilli prononce une phrase sur l'haleine salée du vent qui appartient aussi au révérend (pour ceux qui se rappellent du roman) il reste une ombre de doute sur l'identité de Stevens-Stevens : « Me voir comme j'ai toujours voulu me voir » donne une licence interprétative onirique à la dérive disparate des éléments constitutifs des personnages: «Olivia... belle comme une île. L'île est morte. »

La convergence disparate d'éléments culturels anglais et français prêtait aux Fous de Bassan un flou constitutionnel de superposition des cultures anglophones d'Angleterre et des Etats-Unis, de France et du Québec, dans une position caractéristique des réseaux culturels de l'immigration d'Amérique du Nord et du bilinguisme canadien. L'effet de réversibilité des « traduction » des Anglophone s'exprimant en français permettait un sens de côtoiement de l'anglais au français - alors qu'au retour de traduction du texte original en anglais, l'anglais ne peut guère cerner ni sertir l'anglais. Ce qui entraine un manque de discernement entre les apports homogènes et hétérogènes des cultures qui traversaient le texte des Fous de Bassan de façon plus perceptible. Par contre, dès son titre, In the Shadow of the Wind insiste sur l'impression que le temps d'un cillement, les colonies d'oiseaux et de villageois exposés aux vents marins seront les agents de leur apparition-disparition réversible. Dans un souffle, la généalogie Brown-Jones surgit de Griffin Bay puis s'envole vers le Sud, entre ciel et mer attestant d'une mythologie de colonie (de villageois anglophones, francophones ou de fous de Bassan. L'aspect de migration-immigration originelle de la communauté coloniale représentée par les oiseaux du titre français est subtilement accentué de cette façon.

L'analyse très ponctuelle que l'étude présente a fait de l'excellente traduction de Fischman révèle qu'à l'occasion, une référence shakespearienne est gommée, et le passage à l'anglais occulte certaines dichotomies franco-anglaises, les insertions en anglais dans le texte, et escamote en partie la disparité Nord-Sud dans sa dimension politico-économique 
québécoise à un moment clé du texte. Quelques couleurs nécessaires au système de l'expression des tensions internes entre les personnages sont subsumées par l'utilisation de l'anglais débarrassé du filtre français.

La distorsion de la mémoire onirique contre le paysage comme milieu réaliste-réel est plus sensible dans le film qui révèle une possibilité d'interprétation Romantique de l'action et des ruines qui conservent la trace d'un asile et des vestiges de vies. Le film capitalise sur les espaces physiques et psychologiques internes-externes annonçant les métamorphoses et les étapes de l'intrigue en s'attardant sur les cadrages significatifs des baignoires, sur les trouées des portes et des fenêtres, sur les portraits sans cadres, les plans fixes en clichés de visages, et sur les seuils internes-externes, familiers-étrangers, centrifuges-répulsifs.

Le film exprime un effort de délimiter ou cerner les causes-conséquences des gestes, par exemple en marquant les moments sur lesquels l'action pivote par des insertions de plans sur les oiseaux, de rires qui s'apparentent aux animaux aux moments dramatiques de l'action. Il sertit les quelques mots anglais de Stevens dans le français de la communication des villageois comme les enjeux de l'antagonisme sédentaires et migrateurs. Une des forces de la traduction filmique est de mettre en évidence que la communauté anglophone qui s'exprime en français marque une méfiance envers Stevens parce qu'il revient d'un voyage en rapportant des mots anglais. Le statut indécis du « cousin-voyageur » le destine au seuil des portes ou aux marges des bâtiments qui l'accueillent à son retour. En termes d'identité, de responsabilité, de culpabilité, ou de motivation, Stevens devient un principe étranger disparate facteur d'attraction et de répulsion, parfois dans un même mouvement, pour les villageois qu'il révèle à eux-mêmes, sous l'effet du vent dont il semble devenir l'agent de destruction.

Les fous de Bassan d'Anne Hébert invoquent Shakespeare, Faulkner, Giraudoux, Césaire qui délimitent les référents culturels anglophones et francophones de l'espace bilingue du Canada. Ce côtoiement, qui tend à s'évanouir de la traduction anglaise monolingue, se polarise en une structure oppositionnelle de sédentarité-migration dans l'interprétation filmique de Simoneau. La «traduction » telle que définie par ce roman d'Anne Hébert s'avère être idéalement un type de jointure disparate et mobile où l'identique est pénétré d'une dynamique d'altérité, et vice versa.

\section{Servanne Woodward University of Western Ontario}

